\title{
Very slow positive muons
}

\author{
Morenzoni Elvezio \\ Paul Scherrer Institute, CH-5232 Villigen PSI, Switzerland
}

11 October 1991

\section{Introduction}

Positive and negative muons beams in the energy range between a few $\mathrm{eV}$ and a few tens of $\mathrm{keV}$ and with good phase space properties have been on the wish list of scientists involved in muon physics for many years. All fields of muon science, which, as shown by the large spectrum of contributions to this volume, range from chemistry, condensed matter-, molecular-, atomic- to nuclear- and particle physics, would profit from such a development. Very slow polarized muons would also offer a new tool for surface physics, thin-film and interface studies.

In this paper we will concentrate on the most promising developments for the production of slow and very slow polarized positive muons. Negatively charged muons and their deceleration to few tens of $\mathrm{keV}$ are discussed in another contribution to this volume [1]. The paper is organized as follows. In the next section a short overview of the various efforts toward low energy $\mu^{+}$'s is given. In Section 3 the moderation of $\mathrm{MeV} \mu^{+}$'s to $\mathrm{eV}$ energies is reviewed. Open questions and prospects offered by this technique in relation with the current improvement program and plans at PSI are discussed. Characteristics of a possible tertiary beam of very slow $\mu^{+}$'s are presented in section 4 . In the spirit of the title of this volume, some possible future fields of muon physics research which can be addressed with very low energetic $\mu^{+}$'s are outlined in the last section.

\section{Present status}

In principle low energetic $\mu^{+}$'s can be obtained by tuning a surface $\mu^{+}$beam line to lower momenta where the intensity has the well known $p^{3.5}$ momentum dependence [2]:

$N(p)=N_{S} \frac{p^{3.5}}{30^{3.5}} \exp \left(-t / \tau_{\mu}\right)$

( $\mathrm{p}$ in $\mathrm{MeV} / \mathrm{c}$, t: flight time, $\tau_{\mu}$ : muon lifetime). This dependence has been tested down to $3 \mathrm{MeV} / \mathrm{c}$ [3]. Assuming an intensity at the surface edge $N_{S}$ of $10^{7} \mu^{+} / \mathrm{sec}$ the formula predicts only $\sim 20 \mu^{+}$'s $/ \mathrm{sec}$ at $5 \mathrm{keV}$. The intensity will be even lower in fact, since deflecting and focussing elements of present surface muons beam lines are not designed for such low momenta. The background level from positron contamination would be a disturbing factor for most experiments also.

These limitations led to various proposals of deceleration techniques during the last decade. The production of a low energy muon beam is intimately related to the increase of phase space density. Electronic and stochastic cooling which have been applied successfully to beams of protons, antiprotons and heavy ions to improve the beam quality, are too slow for unstable particles like the muons, where rapid action is required within their lifetime. For muons, the application of friction forces in the form of electric fields, for instance, or deceleration in thin foils combined with rf acceleration has been proposed $[4,5]$. A method to handle the time limitation and to achieve phase space compression has been proposed in [6] and prototype studies have been performed at PSI $[7,8]$. The rapid cooling action (within few $\mu s$ ) is obtained by measuring the phase space coordinates of each individual particle and by correcting the values with variable pulsed electromagnetic fields. The application of such a complex procedure to compress a beam from $40 \mathrm{MeV} / \mathrm{c}$ to few eV represents a tremendous challenge for the present state of the technological art, but the method could be used as an improvement stage of a precooled beam. 
A very simple way to act with non-conservative forces on charged particles is to decelerate them in matter or to use the stop-reemission probability from matter for particles. A broad spectrum of slow muons is obtained by slowing down energetic $\mu^{+}$beams in degraders. If the degrader thickness is $\sim 1 / 2$ of the range, about 0.1 $\%$ of an incoming $28 \mathrm{MeV} / \mathrm{c}$ beam and few percents of a $10 \mathrm{MeV} / \mathrm{c}$ will emerge with energies below $20 \mathrm{keV}$ and a roughly flat spectrum [3]. Through the process of electron capture in the last layers of the target foil, a fraction of these muons can form muonium (Mu) [9] or negative muonium ions $\left(\mathrm{Mu}^{-}\right)[10]$ with typically a few $\mathrm{keV}$ of kinetic energy.

Compression of part of the initial spectrum into a narrow band can also be realized.

For instance emission of polarized muonium at thermal energy from a hot $\mathrm{W}$ foil [11] or from $\mathrm{SiO}_{2}$ powders or aerogel $[12,13]$ has been observed after stopping $\mu^{+'}$ 's inside the solid. For $\mathrm{SiO}_{2}$ yields of thermal muonium up to $12 \%[14]$ with respect to the incoming $\mu^{+}$intensity have been reported, whereas for hot foils the muon production efficiency is $\sim 10^{-2}$. The thermalized muonium emitted from $\mathrm{SiO}_{2}$ grains shows a rest polarization of $25.9 \pm 10.1 \%[13,14]$. From a hot foil source one expects a $50 \%$ polarization loss due to the muonium hyperfine oscillations. Thermal muonium can be ionized by lasers [15] and the resultant $\mu^{+}$'s can be used as a source. This approach is being pursued at the Meson Science Laboratory (University of Tokyo) [16]. The beam intensity can be improved by orders of magnitude by placing the set-up for thermal muonium production at the primary proton beam so that protons can interact directly with the foil. This method needs major technical developments but it promises intensities of several $10^{4}$ very slow muons at the MSL or several $10^{5}$ if the system is installed at the facility proposed by the Japanese Hadron Project.

Recently in a pioneering experiment performed at TRIUMF by a Bell Lab-Triumf-UWW collaboration, it has been shown that very slow $\mu^{+}$'s in the $\mathrm{eV}$ energy range are directly emitted from appropriate degrader materials. With a solid Ar layer deposited on a foil, $\sim 2 \cdot 10^{-5}$ of the incoming surface $\mu^{+}$'s are emitted with energies around $10 \pm 15 \mathrm{eV}[17,18]$. This work has been guided by the experience with positrons where moderation of high energy positrons to $\mathrm{eV}$ energies is widely used as a source; the development of low energy positron beams has been crucially dependent upon the discovery and design of efficient $\mathrm{eV}$ positron moderation schemes. Whereas $\mu^{+}$moderation is at its beginning, experience with positrons gives us confidence that this technique could also be used to produce a source of very slow $\mu^{+}$'s. Combining the present known Ar yields with the intensity of $\sim 3 \cdot 10^{8}$ surface $\mu^{+'} \mathrm{~s} / \mathrm{sec}$, which is predicted to be available after completion of the PSI upgrading program, a tertiary low-energy $\mu^{+}$beam line, delivering several thousand $\mu^{+}$'s/sec in the energy range between $\mathrm{eV}$ and some tens of $\mathrm{keV}$ can be envisaged. For this reason a project study has been started at PSI to systematically study and develop this technique.

\section{Muon moderation}

\subsection{Results}

Moderation in solids is routinely used to produce sources of $\mathrm{eV} e^{+}$'s. Hot single-crystal tungsten of high purity, where the $e^{+}$are not trapped in defects and the diffusion length is high, has proven to be a very efficient and practical moderator with an efficiency $\left(N_{\text {out }}(\mathrm{eV}) / N_{\text {in }}\right)$ of up to few part per thousand [19]. The reemission of the implanted slow positrons that have diffused to the moderator surface is due to the negative work function of the positrons in the metal. The energy distribution is narrow and the highest emission energy correlates with the work function. This does not seem to be the only mechanism for $\mathrm{eV} e^{+}$emission. Positrons injected into ionic crystals have been found to be reemitted with kinetic energies peaked at a few $\mathrm{eV}$; the maximum energy is approximately equal to the electron band gap energy [20]. Positrons are reemitted from rare gas solids with kinetic energies less than the inelastic threshold. Solid $\mathrm{Ne}$ gives the best moderation efficiency of $7 \cdot 10^{-3}$ [21].

Some appropriate analogies can be drawn between $e^{+}$'s and $\mu^{+}$'s, but their mass differences implies important differences in the physical properties of $e^{+}$'s and $\mu^{+}$'s in condensed matter [22]. Even at thermal energies the $\mu^{+}$behaves like a well-localized classical particle, whereas for positrons the wave character is dominant. The neutral fractions ( $\mathrm{Ps}$ and $\mathrm{Mu}$ ) have different binding energies and sizes, positron diffusion rates are much greater than those of $\mu^{+}$.

Drawing guidance from the positron results, two experiments have investigated the emission of slow muons from a few selected solids $[17,23]$ by measuring the energy distribution of surface muons transmitted through thin moderating targets with a magnetic spectrometer and a time-of-flight system. With $\mathrm{Cu}, \mathrm{LiF}$ and $\mathrm{SiO}_{2}$ crystals, muons degraded to $\mathrm{keV}$ energies are observed with an efficiency of about $10^{-7}$ (normalized to the incoming $30 \mathrm{MeV} / \mathrm{c}$ particles). In the case of $\mathrm{LiF}$ (and possibly $\mathrm{SiO}_{2}$ ), the time distribution of the degraded muons indicates an additional slower, separated component at the level of $\sim 2 \cdot 10^{-7}$ originating from muons with fractions of $\mathrm{keV}$ energy. The emission of very slow $\mathrm{Mu}^{-}$is also observed with a rate of a few ions per $10^{7}$ incident $\mu^{+}$'s. Thin films of rare gas solids deposited on a substrate have been found to yield very slow $\mu^{+}$'s with an intensity $\left(\sim 2 \cdot 10^{-5}\right)$ of up to two order of magnitude higher than for LiF. The energy distribution of the moderated $\mu^{+}$'s is peaked with a maximum (for Ar and $\mathrm{Xe})$ at very low energy $(\sim 10 \mathrm{eV})$, a width of $\sim 15 \mathrm{eV}$ and a high energy tail falling off monotonically (Fig.1, [18]).

For $\mathrm{Xe}, \mathrm{Kr}$ and $\mathrm{Ar}$ the moderation efficiency is correlated to the band gap energy, which is large for noble elements. With increasing band gap energy, an increase of the $\mu^{+}$'s yields in the $\mathrm{eV}$ region is observed. To explain this behavior it has been suggested that the observed muons are epithermal, thus retaining part of their initial kinetic energy. 

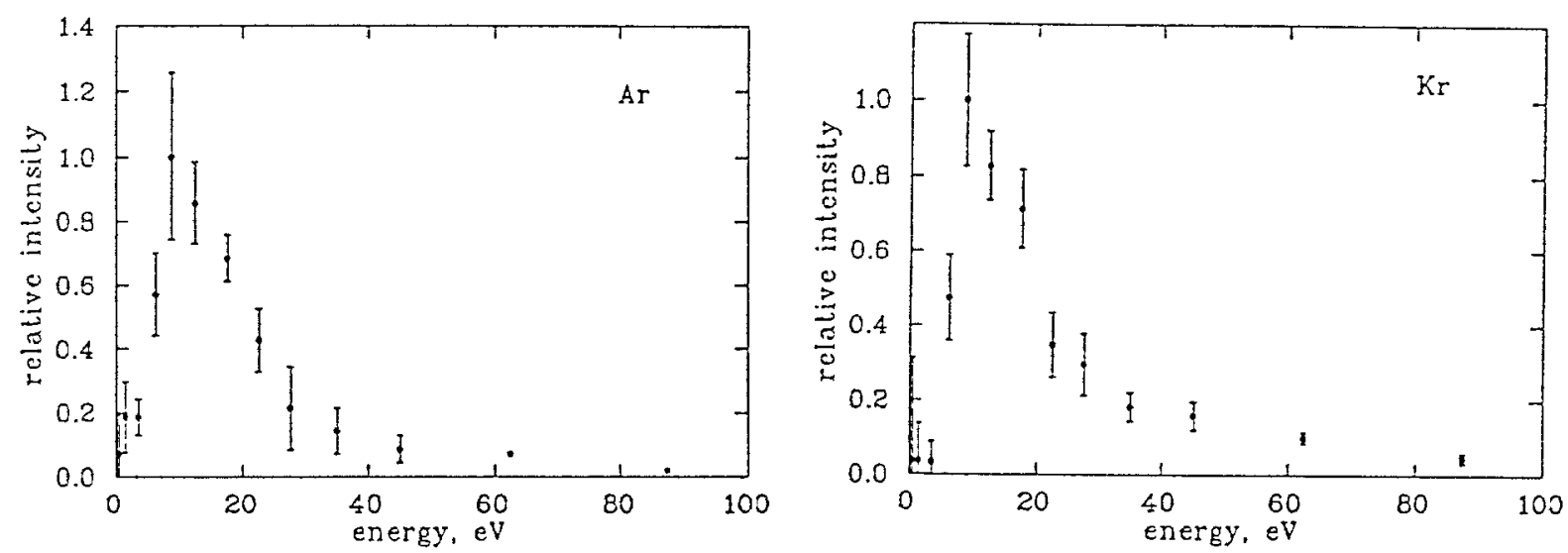

Fig. 1. Energy spectrum of muons moderated in solid Argon and Krypton (from [18]).

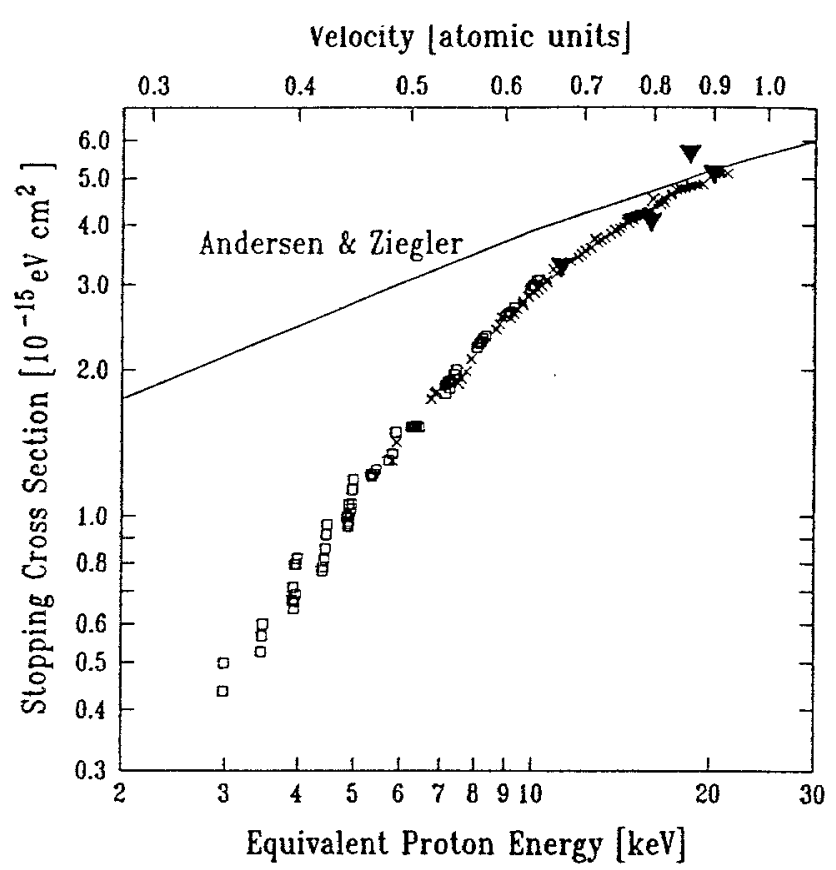

Fig. 2. Stopping cross section of He for protons (cross, triangle) and deuterons (square) [27]. The solid line from [28] shows a linear proportionality below $10 \mathrm{keV}$.

The emission is attributed to the reduction or suppression near and below the threshold of efficient electronic energy lass mechanisms such as electron-hole pair formation $[17,24]$. The decrease in energy loss rate leads to a relatively long escape depth for epithermal muons and therefore to an increased reemission probability. The same mechanism of reduced stopping power was first suggested to explain the positron emission data from alkali halides and rare gas solids [25]. Fermi and Teller [26] were the first to point out that the case of energy loss in insulators differs from that in metals: the amount of energy that may be delivered to a metal is arbitrary small, whereas in insulators it must be at least as large as the gap between two Brillouin zones, typically sev- eral $\mathrm{eV}$. Therefore the loss of energy to electrons will be reduced when energy is transferred in small individual amounts. This effect has been shown very recently in stopping cross section measurements of low energy protons and deuterons in He gas [27] (Fig.2), where the presence of an appreciable minimum excitation energy leads to a significant reduction and to a deviation from the generally assumed linear $v_{p}$ proportionality. A dependence on roughly the third power of $v_{p}$ is found. The threshold effect is appreciable at energies well above the threshold, for protons up to $20 \mathrm{keV}$. Assuming velocity scaling, such an effect would affect muons with less than $2 \mathrm{keV}$ energy.

\subsection{Open questions}

A better understanding of the processes governing the emission of $\mathrm{eV} \mu^{+}$'s from a solid would not only be intrinsically interesting but could be of great relevance for an efficient very slow $\mu^{+}$source. Although the reduced energy-loss mechanism in the few $10 \mathrm{eV}$ region seems plausible, present data do not provide an unambiguous signature about the processes responsible for the production of eV muons. Very little experimental information exists concerning the energy loss of particles with few $\mathrm{keV}$ or less kinetic energy. Recently a formalism was developed to describe the slowing down of $\mu^{+}$'s in gases in terms of the two-body atomic cross sections for target ionization, electron capture, electron loss in muonium and elastic collisions $[24,29,30]$. It predicts the charged and neutral fractions as a function of pressure at various stages of the slowing down process, as well as the polarization.

No similar calculations exist for the solid state. In condensed matter, the mean free path of a muon can be of the same order of magnitude as the atomic dimensions. This means that a muon cannot interact with one atom before coming under the influence of the next. A calculation of the slowing down based on atomic cross sections, allowed at high velocities where the cross sections are small, becomes therefore doubtful at low velocities. Col- 


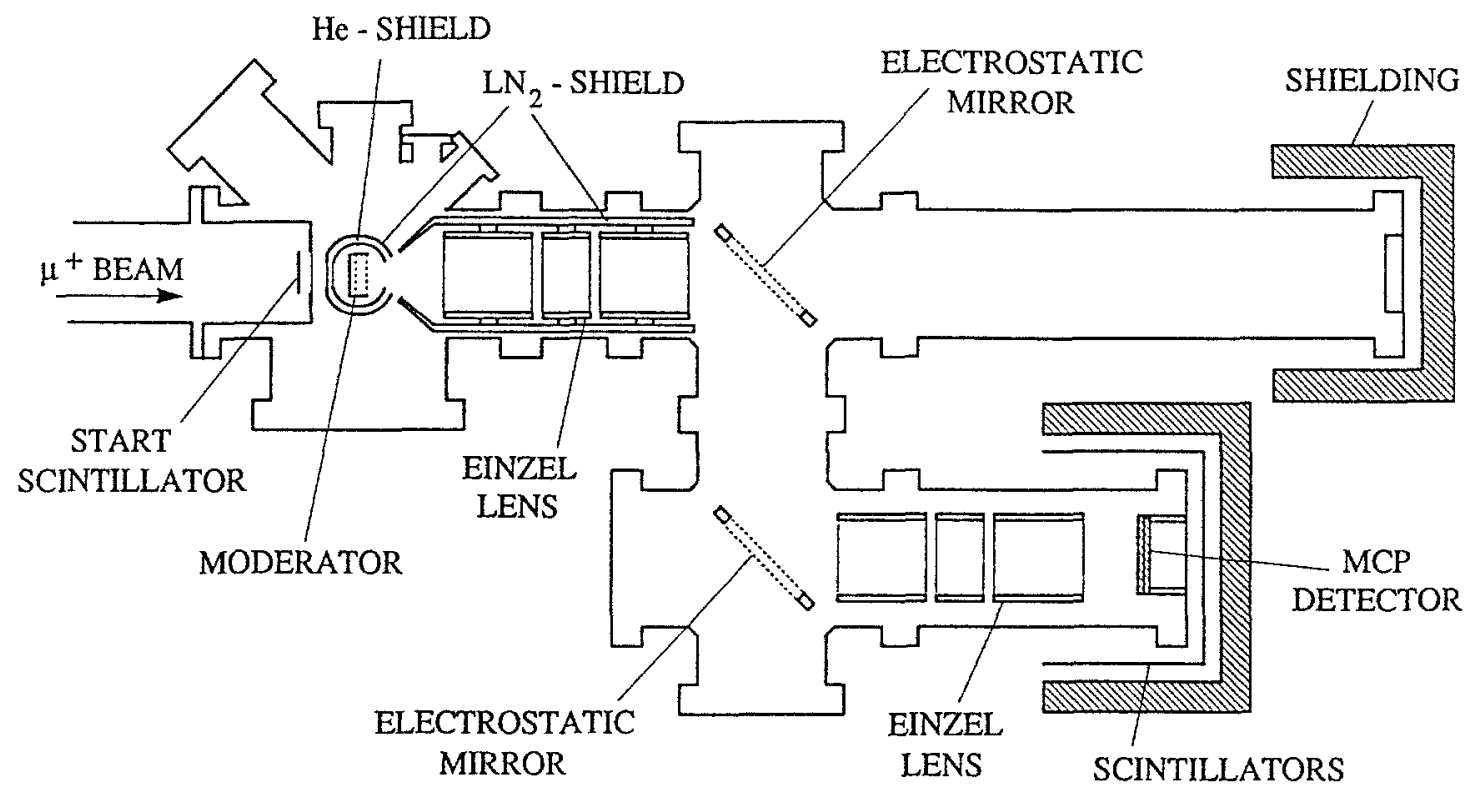

Fig. 3. Setup for the study of slow and very slow muons at PSI. For details see text.

lective effects associated with the solid state and possibly phonon interaction must be taken into account, also.

For the practical purpose of developing a source of very slow muons, the relevant parameters governing the yields and characteristics of the emitted particles must be determined.

The large difference in yields from $\mathrm{LiF}$ and $\mathrm{Ar}$ (about two orders of magnitude) in spite of almost equal band gap energies (13.7 eV for $\mathrm{LiF}$ and $14 \mathrm{eV}$ for Ar) may indicate that this quantity is not the only one of relevance. A similar question arises when comparing the smaller moderation efficiency $\left(\sim 10^{-6}\right)$ of $\mathrm{Ne}\left(\mathrm{E}_{g}=22 \mathrm{eV}\right)$ with that of Ar. Most probably the Ne measurement has been influenced by nonideal experimental conditions.

The experience with low energy $e^{+}$sources shows that only systematic and intense development studies together with technological improvements can exploit the potential of the moderation technique. The first measurement of slow $e^{+}$emission reported an efficiency of $\sim 10^{-7}[31]$. In the meantime the best value is in the percent region [21]. For various physical reasons, an improvement of many orders of magnitude cannot be expected for muons, but it is reasonable to assume that the ultimate value in efficiency has not yet been reached.

\subsection{PSI Plans}

The apparatus shown in Figure 3 is under construction at PSI. Its purpose is to study and optimize the emission of slow and very slow muons from solids and to perform first experiments. It will also serve as a basis for a tertiary beam of very slow muons.

The setup consists of a Vltra High Vacuum chamber with a design vacuum of $10^{-10}$ Torr. In a built-in UHV He-Cryostat, a thin layer of rare gas solids will be formed by vapor deposition on appropriate substrates. To yield a stable Ne film and low vapor pressure, the target will be cooled at liquid helium temperature. The thickness of the layer will be measured with a cooled quartz crystal. UHV conditions are needed to reduce the influence of target contamination on the moderation efficiency. With a vacuum between $5 \cdot 10^{-8}$ and $5 \cdot 10^{-9}$ Torr, a reduction of the Ar yields with time after the deposition was reported in ref. [17]. Recent investigations of slow proton reemission from rare gas solids indicate that the quality of the solified layer plays an important role [32]. This implies that the yields from carefully prepared rare gas solids could be significant better than the reported 2 . $10^{-5}$. With a basis vacuum of $10^{-10}$ Torr, the formation time for a monolayer is of the order of hours, which is a sufficiently long time to provide stable conditions during a measurement.

The yields may depend not only on target composition but also on its structure. Thin films of rare gas solids prepared on a low temperature substrate are polycrystalline with a grain size of a few times the film thickness. Grain size and imperfections may influence the yields especially if diffusion processes are involved [33].

Another aspect to be investigated is possible channeling effects of epithermal muons in crystals of rare gas solids, which could result in a higher efficiency. Thin crystals can be epitaxially grown on single-crystal substrates and their structure has been studied by using low energy electron diffraction [34]. Spare ports in the UHV chamber are foreseen for future implementation of surface and structure analysis instrumentation. Instead of the cryostat, a target ladder could be mounted to study different crystal moderators.

Yields and energy spectra of the $\mathrm{eV} \mu^{+ \text {'s }}$ will be measured with a time-of-flight (TOF) system. The inci- 
dent polarized surface muons traverse a thin scintillator starting the TOF measurement. Part of the muons are moderated in the target, which is electrically isolated to accelerate and extract the slow and very slow muons from the production region.

They are then focussed an a microchannelplate detector (giving the stop signal of the TOF measurement) with a system of einzel lenses and electrostatic mirrors. A second electrostatic mirror is foreseen to reduce the background due to the scattering of higher energy particles off the grid in the first one. In a short section of the TOF, the muons will be slightly accelerated in order to broaden the TOF spectrum and to determine accurately the energy distribution. Alternatively, an integral measurement of the energy spectrum can be performed by putting a grid to a higher voltage than the target. Formation of $\mathrm{Mu}^{-}$can be determined by reversing the voltage polarities. The measurement of the fraction of slow muonium and $\mathrm{Mu}^{-}$relative to $\mu^{+}$as a function of the energy should also help to elucidate the moderation mechanisms.

A very important characteristics is the polarization of the slow muons. In a solid, the total time for the $\mu^{+}$ to decelerate to few eV is typically $10 \mathrm{ps}$. This is sufficiently rapid to avoid any significant loss of polarization during the stopping process even if there is depolarizing muonium formation. In the absence of a delayed emission mechanism, as in the case for epithermal muons, no depolarization is expected. For many applications the muon beam has to be polarized and the polarization must be known. It can be measured by measuring the $\mu^{+}$spin precession signal of muons implanted in a target for which the $\mu S R$ signal is well known.

A complementary approach to the use of solid target moderators is the use of superfluid helium [35]. Here two questions are of relevance. First, is the mechanism of reduced stopping power near the threshold energy effective in superfluid helium too?. If yes, a superfluid helium target (eventually in the form of a thin film) could be used as a moderator. If the ionization energy is the relevant parameter $(\sim 25 \mathrm{eV}$ in $\mathrm{He})$, the efficiency is expected to be higher than with solid rare gases. The second question is whether it is possible to extract stopped $\mu^{+}$'s from superfluid helium. In a $\mu S R$ experiment with liquid helium, it was found that an applied external electric field strongly reduces the relaxation of stopped muons [36]. Moreover in electric fields, with $E>600 \mathrm{~V} / \mathrm{cm}$ muons move with velocities of about $50 \mathrm{~m} / \mathrm{s}$. Krasnoperov [35] proposed to stop a surface $\mu^{+}$beam in superfluid helium at a temperature of $0.5 \mathrm{~K}$. Within 100 ps the muons are thermalized. By applying an electric field perpendicular to the surface, muons drift to the surface. They can be extracted from the surface with a short electric pulse. This method may be efficient since the escape depth may be of the order of $1 \mu m$ due to the high mobility in the electric field. Positive muons attract clusters of helium atoms around them (snowballs) [37]. The question is whether the $\mu^{+}$'s can tunnel through the snowball and be extracted as free $\mu^{+}$'s or whether they leave the surface as $\mu^{+} H e_{n}$ clusters. For an assessment of the fea- sibility if this proposal, the kinetics and the behaviour of $\mu^{+}$in superfluid Helium will be investigated at PSI [38]. The motion of the particles through the liquid vapor interface and the existence of an energy barrier for leaving the liquid helium surface will be also studied.

\section{Tertiary muon beam}

The development program at PSI has been prompted by the upgrading program presently under way. A gradual increase of the proton current to its maximum value of $1.5 \mathrm{~mA}$ by 1994 is being pursued. The new large acceptance $\pi E 5$ beam will be installed beginning of 1992 [39]. At the maximum current, $\pi E 5$ is designed to deliver $3 \cdot 10^{8}$ surface $\mu^{+} / \mathrm{s}$ (Fig. 4). Taking the present (not optimized) moderation efficiency for Ar, a source of $\sim 6000 \mu^{+} / \mathrm{s}$ with energies of $10 \pm 8 \mathrm{eV}$ can be provided by installing a moderator setup in the $\pi E 5$ beam line. At other beam lines, several hundreds $\mu^{+} / \mathrm{s}$ can be delivered.

The moderator represents the source of a tertiary beam line of polarized muons in the energy range between $\sim 10 \mathrm{eV}$ and several tens of $\mathrm{keV}$. Besides an optimized moderator geometry, such a tertiary beam consists of the following elements:

1) A beam handling system to extract the moderated $\mu^{+}$'s from the production target and to separate them from background particles and high energy $\mu^{+}$.

2) A section for tuning the energy. For fast timedifferential measurements, a thin foil detector providing a clean start signal could be inserted in this section. The energy spread introduced by the measurement can be actively compensated with a pulsed electrode.

Since the initial energy spread of the source is small, good energy resolution, determined essentially by the transport and acceleration-deceleration system, can be achieved. The source dimension is determined by the size of the secondary beam. The size of the $\pi E 5$ beam is $4 \mathrm{~cm}$ diameter; a smaller beam spot down to few $\mathrm{mm}$ squared is available at other beam lines at the expense of intensity. Since the very slow muons are accelerated at the target, they emerge with practically zero divergence. According to the Liouville theorem, the $\mu^{+}$'s can be focussed on a small spot whose size is determined only by the transport system.

3) The last part of the facility is a UHV chamber where the various experiments can be accommodated. The moderator chamber, which is also a UHV chamber, is easily compatible with UHV conditions, the preferred medium for applications of very slow muons to surface studies.

A tertiary beam of this type could also be installed in a beam line of a pulsed machine or behind a surface $\mu^{+}$storage ring of the type proposed in [40].

A drastic increase of intensity of this tertiary beam line can be achieved by decelerating a surface $\mu^{+}$beam in the cyclotron trap and subsequently moderating the $\mu^{+}$'s extracted with $\sim 20 \mathrm{keV}$ energy [1]. The moderator efficiency is essentially determined by the ratio $\lambda / \Delta R$ 


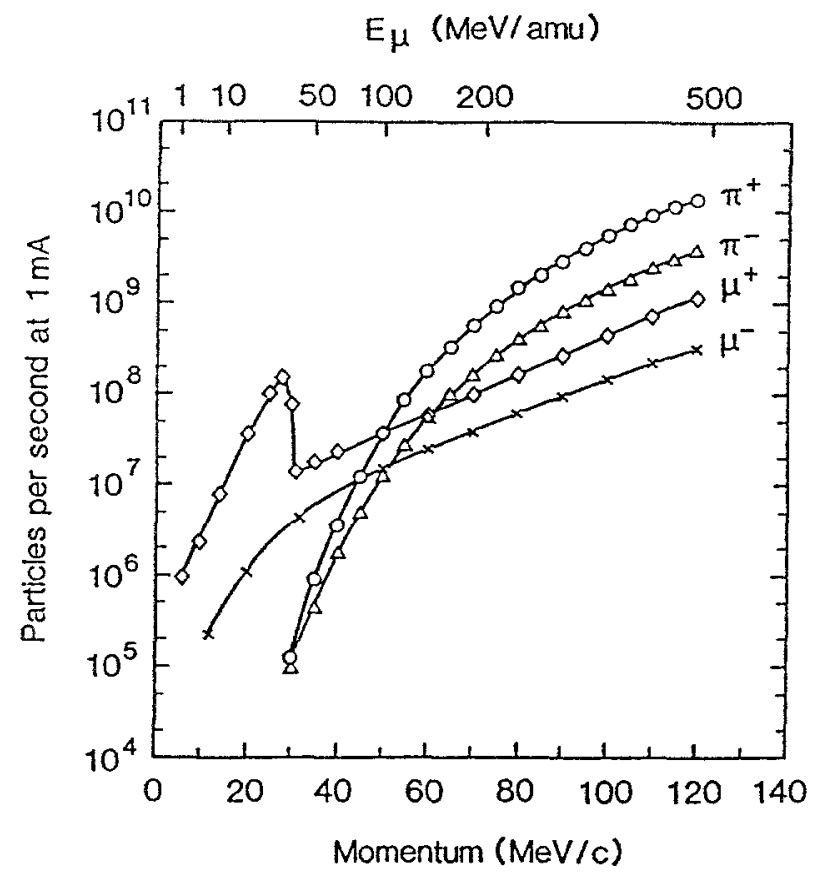

Fig. 4. Intensity of secondary beams at the $\pi E 5$ beam line at PSI with $1 \mathrm{~mA}$ primary proton beam.

( $\lambda$, escape depth of $\mathrm{eV}$ muons; $\Delta R$, stop width of the implanted muons). $\Delta R$ is proportional to $p^{3.5}$; therefore a reduction of the muon momentum prior to their implantation increases the moderation efficiency and the absolute number of epithermal muons. With a combined deceleration and extraction efficiency of $\sim 3 \cdot 10^{-3}$, a factor 40 improvement in $\mathrm{eV}$ yields with respect to the surface $\mu^{+}$intensity is expected. Possible $\mu^{+}$depolarization effects during the deceleration process in the cyclotron trap have to be studied in detail.

\section{Future in muon physics}

A tunable beam of polarized $\mu^{+}$with energies between some $\mathrm{eV}$ and some tens of $\mathrm{keV}$ with intensities of a few thousand per second offers a new powerful instrument for studies in fundamental muon physics as well as for new applications in surface and solid state physics. Without being exhaustive, we list some examples.

With a fine tuning of the $\mu^{+}$energy, the cross sections of basic atomic processes (ionization, electron capture, muonium ionization, scattering, energy loss in matter) can be obtained as a function of the energy. By comparing these results with those obtained with proton or hydrogen beams, mass effects and the velocity scaling of the cross sections can be tested. Related cross sections in $\mu^{+}$-molecule collisions and molecular ion formation can be investigated as well. A detailed understanding of the fundamental interactions of very low energy $\mu^{+'}$ 's with matter is also of importance for an optimization of the moderation method. Slow and very slow $\mu^{+}$'s have an important impact in muonium production and muonium physics. A muonium beam with tunable energy can be formed by passing low energy $\mu^{+}$'s through a thin foil or gas target, where the $\mu^{+}$'s are neutralized with large probability [3]. The energy straggling can be kept small. With an appropriate target, the neutralization can result preferentially in excited states. For instance, from low energy collisions (below a few $\mathrm{keV}$ ) of proton on $\mathrm{Cs}$, it is known that practically all electron pick-up populates $n=2$ states. Up to $40 \%$ of those atoms are in the metastable 2s state [41]. Muonium in the 2s state, a preferred system for precision spectroscopy [42], could be formed this way.

With a low energy beam of $\mu^{+}$'s, a detailed study of thermal Mu production in hot foils or Silica grains can be performed. On the other hand, by implanting $\mathrm{keV}$ or sub keV $\mu^{+}$'s into such surfaces an efficient and clean thermal $\mathrm{Mu}$ source is obtained.

A beam of tunable low energy polarized $\mu^{+}$'s provides a new tool for surface physics also. A polarized $\mu^{+}$is a natural candidate to study surface, thin-film and interface magnetism, subjects of considerable relevance to both fundamental research and to applications. New techniques such as scattering of $\mu^{+}$'s on surfaces or the application of $\mu S R$ type measurements to surfaces, thin films and interface studies could be developed. The $\mu S R$ method is now limited to the study of bulk characteristics of the solid state, because the surface muons generally used have a typical penetration depth of some tenths of millimeter with a stopping distribution of comparable width. With a tunable beam of a few $\mathrm{keV}$, the surface and thin film regions become accessible. The accessible range can be estimated by scaling the scarce proton results [43]: one expects for a $100 \mathrm{eV}^{+}$a range in the order of $10 \AA$ and for a $10 \mathrm{keV}$ a range of the order of $1000 \AA$.

Detailed studies of the interaction of the very slow muons when the particle reaches the surface of a solid from outside (stopping profile, neutralization, reemission probability..) are needed in order to fully exploit the potential. At relatively high velocities (a few $\mathrm{keV}$ ), the dominant process is probably deep implantation and full thermalization. At lower energies, the mean implantation depth becomes so small that a non negligible fraction comes back to the surface before being thermalized. Moreover, passing through the surface, the $\mu^{+}$can bind one or two electrons and emerge as $\mathrm{Mu}$ or $\mathrm{Mu}^{-}$.

Magnetic order in the topmost layer could be studied by scattering slow $\mu^{+}$'s at grazing incidence also. Spin polarized electrons at the surface are captured by polarized $\mu^{+}$'s. If the electron spins are parallel to the muon spin, only muonium in the triplet state is formed and the muons maintain their polarization. If the electron spins are antiparallel, the $\mathrm{Mu}$ is in a $50 \%$ triplet and a $50 \%$ singlet combination and the $\mu^{+}$depolarizes due to the hyperfine interaction. Therefore, by measuring the $\mu^{+}$ polarization, the electron spin polarization can be determined. Analogue experiments have been proven feasible with deuterons [44] and polarized positrons [45]. Surface studies can also be performed with muonium such as investigations of the electronic structure around $\mathrm{H}$-like 
impurities on surfaces, of catalytic chemical reactions of Mu on metal surfaces, or of Muonium hyperfine field on surface.

\section{Conclusions}

Due to its own production mechanism, the muon has a large kinetic energy initially and a large spread in energy. Despite their energetic appearance, muons have found many applications down to thermal energies.

A new dimension in muon physics would be opened with the availability of tunable beams of polarized muons in the energy range between some $\mathrm{eV}$ and few tens of $\mathrm{keV}$.

Much work remains to be done, but the continuous progress of experimental techniques and machines providing us with muons make us confident that this goal can be reached.

To paraphrase V. Hughes and G. zuPutlitz, to whom this volume is dedicated, muons and muonium have not yet decayed [46].

Acknowledgement. Stimulating discussions with J. Beveridge, K. Jungmann, M. Meyberg and L. Simons are gratefully acknowledged.

\section{References}

1. L. Simons, this volume

2. A. E. Pifer et al. Nucl. Instr. and Methods 135, 39 (1976)

3. A. Badertscher et al., Nucl. Instr. and Meth. A238 200 (1985)

4. H. Daniel, Z. Phys. A313, 249 (1983)

5. H. Daniel, Muon Cat. Fusion 4, 109 (1989)

6. D. Taqqu, Nucl. Instr. and Methods A 247, 288 (1986)

7. A. Fuchs, E. Morenzoni, E. Pedroni, D. Taqqu, to be published

8. L. Simons, E. Morenzoni, F. Kottmann in Electromagnetic cascades and chemistry of exotic atoms, Ettore Majorana Institute (1989), Erice, Science Series, Phys. Sciences, Vol 52.

9. P.R. Bolton et al, Phys. Rev. Lett. 47, 1441 (1981)

10. Y. Kuang et al., Phys. Rev. A60, 3172 (1987).

11. A. P. Mills Jr. et al., Phys. Rev. Lett. 56, 1463 (1986)

12. G. A. Beer et al., Phys. Rev. Lett. 57, 671 (1986)

13. W. Schäfer, Ph. D. Thesis, University of Heidelberg, 1988

14. K.A. Woodle et al., Z. Phys. D9, 59 (1988).
15. S. Chu et al., Phys. Rev. Lett. 60, 101 (1988).

16. K. Nagamine, Hyperfine Interactions 65, 1149 (1990).

17. D.R. Harshman et al., Phys. Rev. B36, 8850 (1987)

18. G. D. Morris, Master of Science Thesis, University of British Columbia, 1990.

19. P.J. Schultz and K.J. Lynn, Rev. of Mod. Phys. 60, 701 (1988) and ref. therein

20. A. P. Mills Jr. and W. S. Crane, Phys. Rev. Lett. 53, 2165 (1984).

21. A. P. Mills Jr. and E. M. Gullikson, Appl. Phys. Lett. 49, 1121 (1986).

22. F. M. Jacobsen, Hyperfine Int. 32, 501 (1986).

23. D.R. Harshman et al., Phys. Rev. Lett. 56, 2850 (1986)

24. M. Senba, J. Phys. B 21, 3093 (1988).

25. K.G. Lynn and B. Nielsen, Phys. Rev. Lett. 58, 81 (1987)

26. E. Fermi and E. Teller, Phys. Rev. 72, 399 (1947)

27. R. Golser, D. Semrad, Phys. Rev. Lett. 66, 1831, (1991).

28. J.F. Ziegler, J.P. Biersack, U. Littmark, The Stopping and Range of Ions in solids, Vol. 1, Pergamon Press 1985.

29. M. Senba, J. Phys. B 22, 2027 (1989).

30. M. Senba, J. Phys. B 21, 1545 (1990).

31. W. Cherry, Ph. D. Thesis Princeton University 1958.

32. A.P. Mills Jr. et al., Phys. Rev. B42, 5973 (1990).

33. J. A. Venables and B. L. Smith, in Rare gas Solids, Vol. II, M. L. Klein and J. A. Venables editors, Academic Press, 1977.

34. H.H. Farrel et al., Phys. Rev. B6, 4703 (1972).

35. E.P. Krasnoperov, Hyperfine Interactions, 65, 1049 (1990)

36. D. G. Eschenko et al., JETP Lett., 48, 616 (1988).

37. K.R. Atkins, Phys. Rev. 116, 1339 (1959).

38. E.P. Krasnoperov et al., PSI Proposal, Nov. 1990.

39. D. Renker, this volume.

40. J. Brewer, Hyperfine Interactions, 65, 1137 (1990).

41. V.N. Tuan, G. Gautherin, A.S. Schlachter, Phys. Rev. A49, $1242(1974)$.

42. K. Jungmann, this volume.

43. G. G. Ross and B. Terreault, Nucl. Instr. and Methods B15, 61 (1986).

44. C. Rau, R. Sizmann, Phys. Lett. 43A, 317 (1973).

45. D. W. Gidley et al., Phys. Rev. Lett. 24, 1779 (1982).

46. V. Hughes and G. zuPutlitz, Comm. in Nucl. and Part. Physics, 2, 259 (1984).

This article was processed using Springer-Verlag TEX Z.Physik C macro package 1991

and the AMS fonts, developed by the American Mathematical Society. 